\title{
Enhanced uptake and transport of PLGA-modified nanoparticles in cervical
} cancer

Lee B. Sims ${ }^{1 \dagger}$, Louis T. Curtis ${ }^{1 \dagger}$, Hermann B. Frieboes ${ }^{1,2,3 \neq}$ and Jill M. Steinbach-Rankins ${ }^{1,3,4,5^{*} \neq}$

\begin{abstract}
Background: Uncoordinated cellular proliferation and dysregulated angiogenesis in solid tumors are coupled with inadequate tissue, blood, and lymphatic vascularization. Consequently, tumors are often characterized by hypoxic regions with limited access to vascular-borne substances. In particular, systemically administered nanoparticles (NPs) targeting tumor cells and relying on vascular access to reach tumor tissue can suffer from limited therapeutic efficacy due to inhomogeneous intra-tumor distribution and insufficient cellular internalization of NPs. To circumvent these challenges, NP surfaces can be modified to facilitate tumor interstitial transport and cellular uptake.

Results: We create poly(lactic-co-glycolic) acid NPs modified with MPG, polyethylene glycol (PEG), MPG/PEG, and Vimentin (VIM), and evaluate their cellular uptake in 2D (monolayer) cell culture of human cervical carcinoma (HeLa). We compare NP performance by evaluating uptake by non-cancerous vaginal (VK2) cells. We further assess NP interstitial transport in hypo-vascularized lesions by evaluating the effect of the various modifications on NP penetration in $3 \mathrm{D}$ cell culture of the HeLa cells. Results show that after $24 \mathrm{~h}$ incubation with HeLa cells in monolayer, MPG, MPG/PEG, PEG, and VIM NPs were internalized at $66 x, 24 x, 30 x$, and $15 x$ that of unmodified NPs, respectively. In contrast, incubation with VK2 cells in monolayer showed that MPG , MPG/PEG, PEG, and VIM NPs internalized at $6.3 \times, 4.3 \times, 12.4 \times$, and $3.0 \times$ that of unmodified NPs, respectively. Uptake was significantly enhanced in tumorigenic vs. normal cells, with internalization of MPG NPs by HeLa cells being twice that of PEG NPs by VK2 cells. After $24 \mathrm{~h}$ incubation in HeLa 3D cell culture, MPG and MPG/PEGNPs were internalized $2 x$ and $3 x$ compared to PEG and VIM NPs, respectively. Whereas MPG NPs were internalized mostly in the cell culture periphery $(1.2 \times, 1.4 \times$, and $2.7 \times$ that of PEG, MPG/PEG, and VIM NPs, respectively), PEG NPs at $250 \mu \mathrm{m}$ penetrated $2 \times$ farther into the tissue culture than MPG NPs. For all NP types, cellular internalization was severely hindered in 3D compared to monolayer.
\end{abstract}

Conclusions: Although MPG surface modification enhances internalization and uptake in hypo-vascularized cervical tissue culture, coating with PEG reduces this internalization while enhancing penetration. A delivery strategy combining NPs with either modification may balance cellular internalization vs. tissue penetration in hypo-vascularized cervical cancer lesions.

Keywords: Nanoparticles, PLGA, MPG, PEG, Vimentin, Tumor spheroids, 3D cell culture, Cervical cancer, Vaginal epithelium, Cell penetrating peptide

\footnotetext{
*Correspondence: jill.steinbach@louisville.edu

${ }^{\dagger}$ Lee B. Sims and Louis T. Curtis contributed equally to this work

${ }^{\ddagger} H$ Hermann B. Frieboes and Jill M. Steinbach-Rankins joint senior authorship

${ }^{1}$ Department of Bioengineering, University of Louisville, 505 S. Hancock,

CTRB 623, Louisville, KY 40208, USA

Full list of author information is available at the end of the article
} 


\section{Background}

Cervical cancer is the third most common gynecologic cause of cancer associated with patient fatalities. Approximately 13,000 new cases of invasive cervical cancer are diagnosed yearly, of which $30 \%$ prove fatal. In the US, cervical cancer primarily afflicts women younger than 50 ; however, in countries without established screening and prevention programs, cervical cancer remains the second most common type of cancer and cause of death among all female cancers [1-3]. Screening tests and vaccines have contributed to a decrease in cases; to date, there are 3 approved vaccines against cervical cancer. These vaccines, Gardasil ${ }^{\circledR}$, Gardasil $^{\circledR}$ 9, and Cervarix ${ }^{\circledR}$, aim at preventing cancers originating from HPV types 16 and 18, which are attributed to $\sim 70 \%$ of cervical cancers [4]. Yet despite this efficacy, the vaccines only protect against a subset of all known HPV strains [4-6]. The inadequacy of vaccination, coupled with the fact that vaccination is not widespread [5, 6], maintains the risk of cervical cancer as a fatal disease.

Relative to the success of prophylactic vaccines, ineffective treatment options exist for established HPV infections and cervical cancer originating from HPV. This is primarily attributed to multidrug resistance and chemotherapeutic side effects. Despite early stage tumor identification and established eradication methods including surgery and radiation, the adverse side effects of these treatment strategies often result in negative gynecologic and obstetric outcomes. In comparison to surgical and radiation challenges, even systemic chemotherapy results in relatively low transport efficiency, resulting in high chemotherapeutic doses needed to target mucosal and epithelial tissue [7-10]. Due to the high systemic toxicity induced by systemic chemotherapy, new treatment strategies are urgently needed.

In addition to these clinical challenges, successful therapeutic agent delivery to the tumor microenvironment requires minimizing agent degradation and excretion, avoidance of immunogenic interactions, adequate penetration and distribution throughout the tumor tissue, cellular uptake and internalization, and sufficient cytotoxicity [11]. United States Food and Drug Administration (FDA)-approved materials may be selected as nano-scale drug carriers that have been proven to be non-inflammatory and non-toxic while enabling the delivery of highly localized concentrations of both hydrophilic and hydrophobic agents [11-13]. In particular, polymer nanoparticle-based drug delivery systems have been evaluated as attractive options for efficacious delivery of agents such as drugs and genes with resulting treatment efficacy $[12,13]$. The pharmacokinetics of polymeric systems can be tailored by changing the polymer monomer concentrations to facilitate agent diffusive transport, typically via a combination of burst and sustained release profiles.

Besides enabling the sustained release of encapsulated agents, relative to systemic options, local intravaginal therapy may represent a relatively non-invasive approach to locally treat cervical tumors [7]. Nanoparticles (NP) can be modified to minimize unwanted systemic interactions, prolong bioavailability, promote targeted delivery to the physiologic site of interest, and enhance tumor penetration and cellular uptake [14]. Different types of NP surface modifications can be utilized to elicit these desired behaviors. Stealth coatings such as polyethylene glycol (PEG) can decrease unwanted systemic interactions and neutralize the carrier's surface charge $[15,16]$, while targeting ligands can increase the specificity of conjugated carriers to systemically target the desired physiological environment [17]. Cell penetrating peptides, such as MPG, RGD, and TAT, are examples of short ligands that can enhance the cellular internalization of their conjugated carriers [18-29]. In particular, previous studies have shown that NP surface modification with the amphipathic, synthetic peptide MPG derived from HIV gp41 and SV40 [24, 30] displays high cellular binding and internalization achieved via clathrin-mediated endocytotic uptake [31].

Tumor tissue is typically characterized by an overabundance of extracellular matrix as well as poorly vascularized areas, both of which can hinder diffusive transport of NPs [11]. Thus, in addition to evaluating cellular uptake capability, delivery systems would benefit from testing in a three-dimensional (3D) environment which more closely represents hypo-vascularized tumor lesions. For this purpose, 3D cell culture provides a suitable platform [32, 33] that is more controllable than tumors in vivo. Previously, 3D cell culture has been employed to study the behavior of various nano-carriers, such as gold and polymeric NPs. It has been shown both experimentally [34-38] and theoretically [39-49] that NPs require appropriate characteristics and surface modifications to adequately penetrate hypo-vascularized regions for effective therapeutic delivery, even if cellular internalization is optimized.

With the goal to enhance both the transport and internalization of therapeutic agents in hypo-vascularized tumor tissue, in this study we evaluate a variety of poly(lactic-co-glycolic) acid (PLGA) NP formulations for tissue penetration and cellular internalization. The NPs were modified with either a cell penetrating peptide (MPG), a stealth ligand (PEG), a tumor targeting cell penetrating peptide (Vimentin tubulin binding site peptide, VIM) $[50,51]$, or a hybrid modification with both cell penetrating peptides and stealth ligands (MPG/PEG). These ligands were chosen because they have already been individually well characterized to enhance cell internalization, e.g., as we have previously shown for MPG 
[31]. We assess how the NP surface modifications affect uptake in cervical tumor cells, and compare the NP performance in normal vaginal cells. We further evaluate the NP transport and internalization in a 3D spheroid model representing hypo-vascularized cervical cancer lesions.

\section{Results}

\section{Nanoparticle characterization}

The NP surface was first modified with avidin-palmitate, and different NP groups were created based on the addition of one of the following biotinylated ligands: MPG, MPG/ PEG, PEG, or VIM, as described in the "Methods" section. Figure 1 illustrates the NPs used in this study, while Additional file 1: Figure S1 shows a representative scanning electron microscopy (SEM) image of the unmodified NPs and their size distribution. The average unhydrated NP diameter measured $167 \pm 50 \mathrm{~nm}$. Hydrodynamic sizes and surface charges for the different NP groups were quantified via dynamic light scattering (DLS) and zeta potential measurements, respectively (Additional file 1: Table S1).

Unmodified NPs had a hydrodynamic diameter of $267 \pm 13.6 \mathrm{~nm}$ and a negative surface charge of $-17.3 \pm 0.5 \mathrm{mV}$, and the addition of avidin-palmitate increased this surface charge to $-14.3 \pm 0.59 \mathrm{mV}$. The surface charge was further increased with the addition of the ligands, with MPG-modified NPs having the most positive charge at $-0.4 \pm 0.2 \mathrm{mV}$, followed by PEG and MPG/PEG with $-5.2 \pm 1.3$ and $-5.3 \pm 0.7 \mathrm{mV}$, respectively, and VIM with $-11.1 \pm 1.0 \mathrm{mV}$. NP hydrodynamic sizes ranged from 232 to $277 \mathrm{~nm}$ for both unmodified and surface-modified groups, with no statistically significant difference between the groups.

\section{Uptake of PLGA-modified nanoparticles in cervical cancer cell monolayers}

Cellular association (binding plus internalization) and internalization in 2D cell culture monolayers were quantitatively assessed using Fluorescence Activated Cell Sorting (FACS) with cervical tumor epithelial cells
(HeLa) and normal vaginal epithelial cells (VK2) (Fig. 2). Both HeLa and VK2 cells were incubated with the same concentration of NPs $(0.05 \mathrm{mg} / \mathrm{mL})$ for 1.5 or $24 \mathrm{~h}$, regardless of modification. For HeLa cells, all of the modified NP groups showed both greater total association as well as internalization relative to unmodified NPs at both time points. At $1.5 \mathrm{~h}, \mathrm{MPG}, \mathrm{MPG} / \mathrm{PEG}, \mathrm{PEG}$, and VIM NPs were internalized at $607 \times, 184 \times, 79 \times$, and $57 \times$ that of unmodified NPs, respectively (Fig. 2a). Comparing the formulations to each other, the MPG NPs showed $3.3 \times$, $7.6 \times$, and $10.5 \times$ greater internalization than the MPG/ PEG, PEG, and VIM NPs, respectively. After $24 \mathrm{~h}$ of incubation, MPG, MPG/PEG, PEG, and VIM NPs were internalized at $66 \times, 24 \times, 30 \times$, and $15 \times$ that of unmodified NPs, respectively (Fig. 2b). The MPG NPs were internalized at $2.7 \times, 2.2 \times$, and $4.5 \times$ that of MPG/PEG, PEG, and VIM NPs, respectively. Since the internalization for the MPG NPs was essentially the same at both time points, these data highlight the increase in internalization of the other formulations after longer exposure times.

\section{Uptake of PLGA-modified nanoparticles in vaginal epithelial cell monolayers}

In contrast, NP association with VK2 cells at $1.5 \mathrm{~h}$ was not as distinct between the various modified formulations (Fig. 2c), whereas PEG NPs internalized at $2.1 \times$, $5.9 \times$, and $8.8 \times$ compared to MPG, MPG/PEG and VIM NPs, respectively. Comparing to unmodified NPs, the MPG, MPG/PEG, PEG, and VIM NPs internalized respectively at $20 \times, 7 \times, 43 \times$, and $5 \times$. After 24 h however, a clearer separation in association was apparent and PEG NPs were internalized at $1.9 \times, 2.8 \times$, and $4.0 \times$ compared to MPG, MPG/PEG, and VIM NPs, respectively (Fig. 2d).

The internalization remained invariant when comparing MPG and PEG between the two time points, while the internalization of MPG/PEG and VIM essentially doubled. When compared to unmodified NPs after $24 \mathrm{~h}$ incubation, the MPG, MPG/PEG, PEG, and
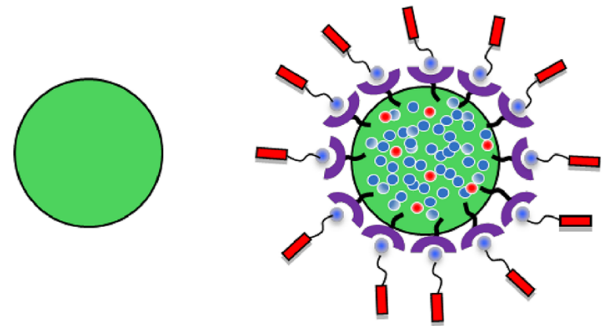

Unmodified
MPG, Vim, or PEG modified

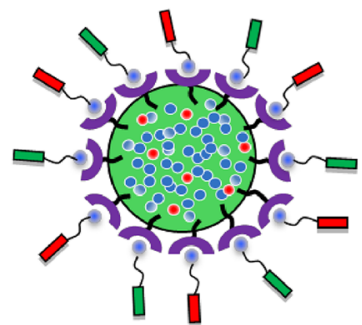

MPG/PEG (Hybrid) modified

Fig. 1 Schematic illustrating NP formulations used in this study 


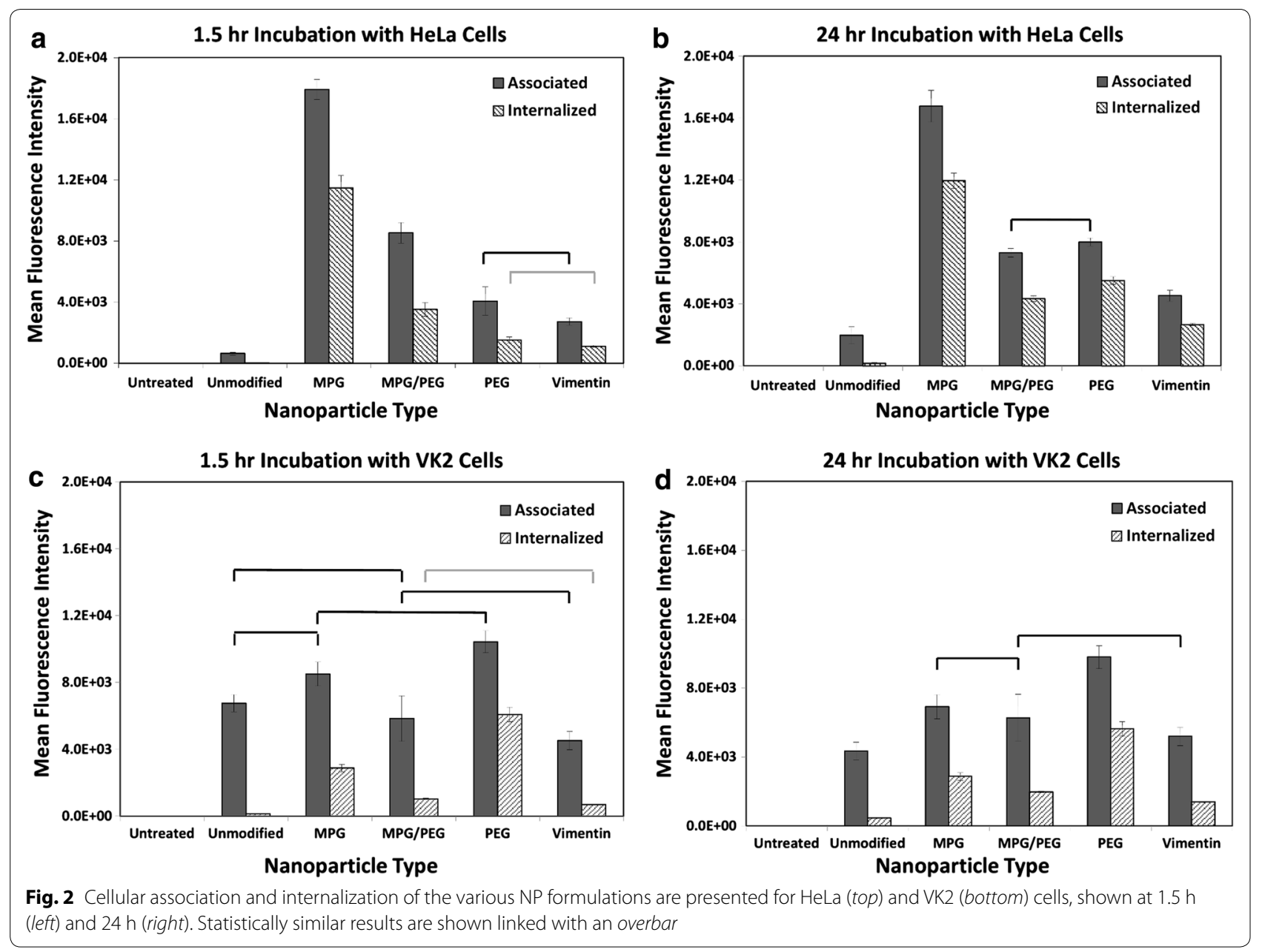

VIM internalized at $6.3 \times, 4.3 \times, 12.4 \times$, and $3.0 \times$ that of unmodified NPs.

\section{Comparison of nanoparticle uptake between tumorigenic and normal cell monolayers}

Of note after $24 \mathrm{~h}$, the unmodified NPs readily associate with the VK2 cells, in contrast to HeLa cells; however, unmodified NPs demonstrate low levels of internalization in both cell lines. In contrast, surface-modified NP uptake was significantly enhanced in both the HeLa and VK2 cell lines. MPG NPs were internalized the most in HeLa cells; whereas PEG NPs were internalized most highly in VK2 cells. While both surface-modified groups (MPG and PEG) demonstrated strong internalization in HeLa and VK2 cells respectively, MPG was internalized in HeLa cells $2 \times$ that of the PEG NPs by the VK2 cells. When comparing between the earlier and later time points, it is apparent that the PEG NPs showed the highest increase in both association and internalization by the longer exposure with the tumorigenic HeLa but not with the normal VK2 cells. Cellular association and internalization were qualitatively assessed via inverted epifluorescence microscopy for both HeLa and VK2 cells at $1.5 \mathrm{~h}$ (Additional file 1: Figures S2, S3) and $24 \mathrm{~h}$ incubation times (Figs. 3, 4). Although the images for the most part reflect the flow cytometry results in Fig. 2, they are representative samples providing a limited assessment.

\section{Uptake of PLGA-modified nanoparticles in hypo-vascularized cancer lesions}

Cellular association and internalization in 3D cell culture (HeLa cervical tumor spheroids), incubated with the same concentration of NPs $(0.05 \mathrm{mg} / \mathrm{mL})$ as the monolayers, were quantitatively assessed at 1.5 and $24 \mathrm{~h}$ via flow cytometry (Fig. 5). At $1.5 \mathrm{~h}$, as a group, all of the modified NPs had higher association than the unmodified NPs, with statistically similar association when compared to each other. The surface-modified NPs also evinced a statistically similar internalization when compared to each other, but in this case, as a group the MPG, MPG/ PEG, and VIM NPs showed higher internalization than the unmodified and PEG NPs (Fig. 5a). In contrast, after 

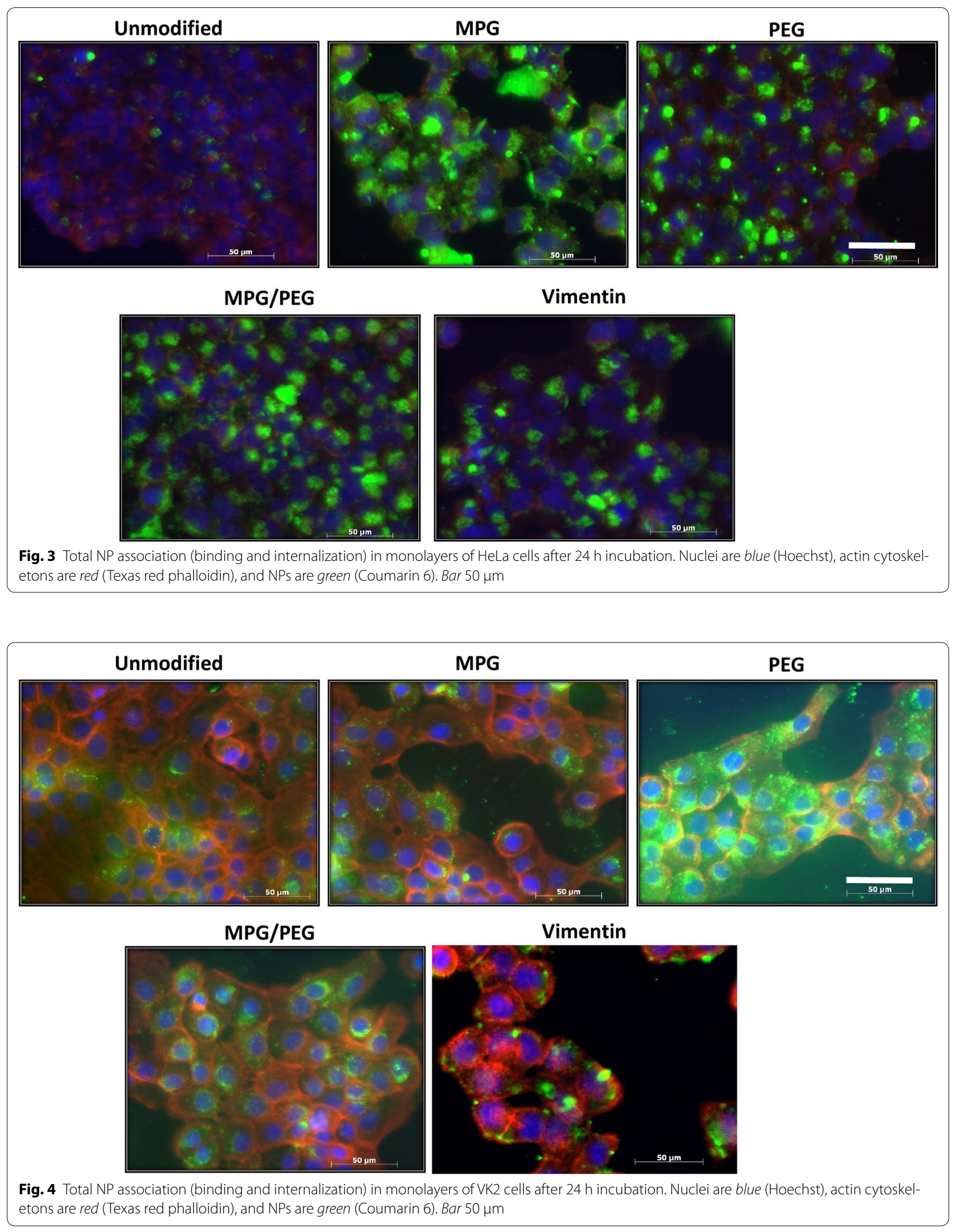

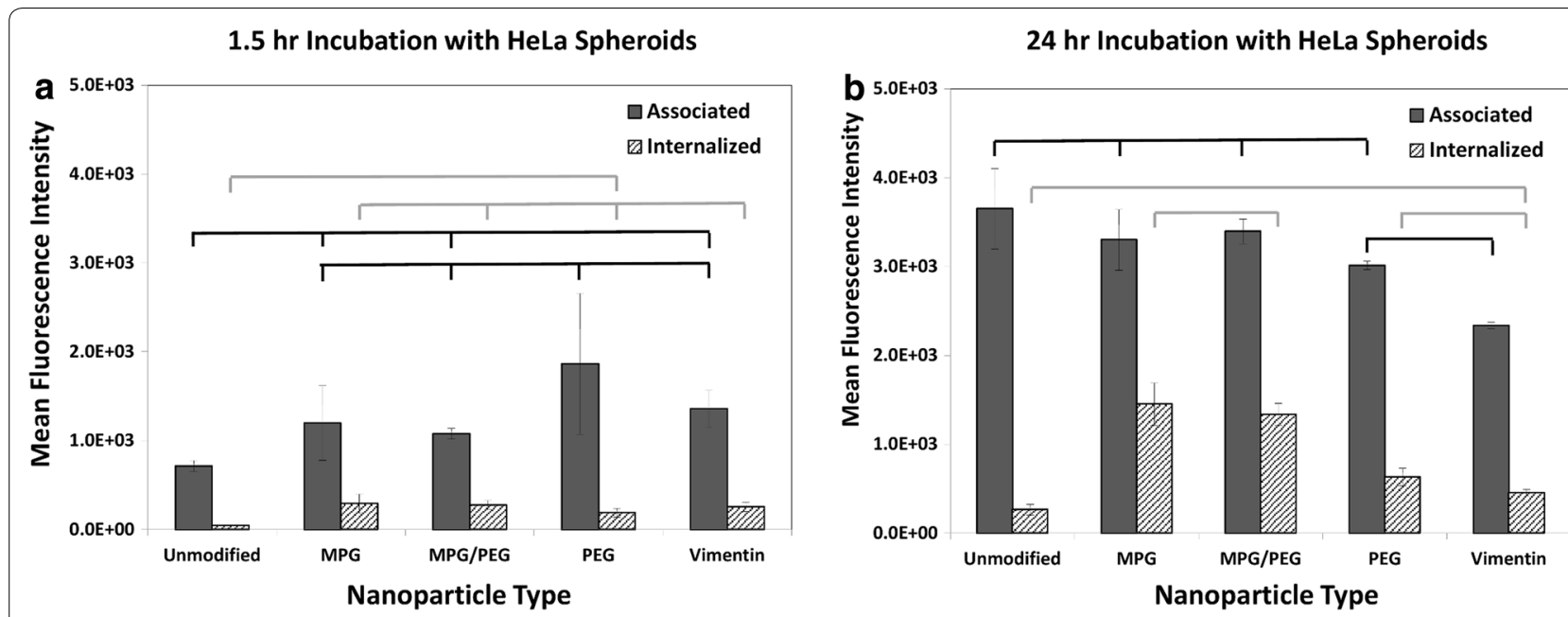

Fig. 5 Cellular association and internalization of the various NP formulations in HeLa cell tumor spheroids. Results are shown at (a) 1.5 and (b) 24 h. Statistically similar results are shown linked with an overbar

$24 \mathrm{~h}$, the unmodified and surface-modified NPs (except for VIM, which was lower) showed statistically similar association (Fig. 5b), while MPG and MPG/PEG showed $2 \times$ and $3 \times$ internalization relative to PEG and VIM NPs, respectively. Interestingly, unlike the results obtained with the monolayer, in the 3D cell culture the association of all NPs increased significantly at $24 \mathrm{~h}$ compared to the $1.5 \mathrm{~h}$ time point: unmodified by $4.8 \times$, MPG by $2.6 \times$, MPG/PEG by $3.0 \times$, PEG by $1.6 \times$, and VIM by $1.6 \times$. Internalization also increased at the later time point, with MPG and MPG/PEG NPs being internalized the most (at $3.6 \times$ and $3.8 \times$ compared to $1.5 \mathrm{~h}$, respectively), while VIM or unmodified versions were internalized the least.

\section{Comparison of nanoparticle uptake between 2D and 3D cell cultures}

In general, all of the values measured from 3D cell cultures were lower than those from the 2D cell cultures, highlighting the effect of the diffusive transport barrier in hypo-vascularized tissue. Figure 6 summarizes the NP cellular internalization as a function of surface modification, cell culture type, and treatment duration, highlighting the effect of diffusive transport on NP uptake between the monolayer and tumor environments. It is expected that monolayer culture represents optimal conditions in terms of diffusive transport, and therefore association and internalization would be lower in the 3D tumor environment.

\section{Transport of PLGA-modified nanoparticles} in hypo-vascularized cancer lesions

The NP diffusion profiles after $1.5 \mathrm{~h}$ through the spheroid tissue are presented in Fig. 7, showing that MPG and PEG-modified NPs exhibited the greatest fluorescence intensity averaged over $200 \times 80 \mu \mathrm{m}$ areas, followed by MPG/PEG and VIM NPs. In particular, MPG NPs were heavily detected in the periphery (within $100 \mu \mathrm{m}$ of the edge) of the spheroids, viz., $2 \times, 1.4 \times$, and $2.7 \times$ that of
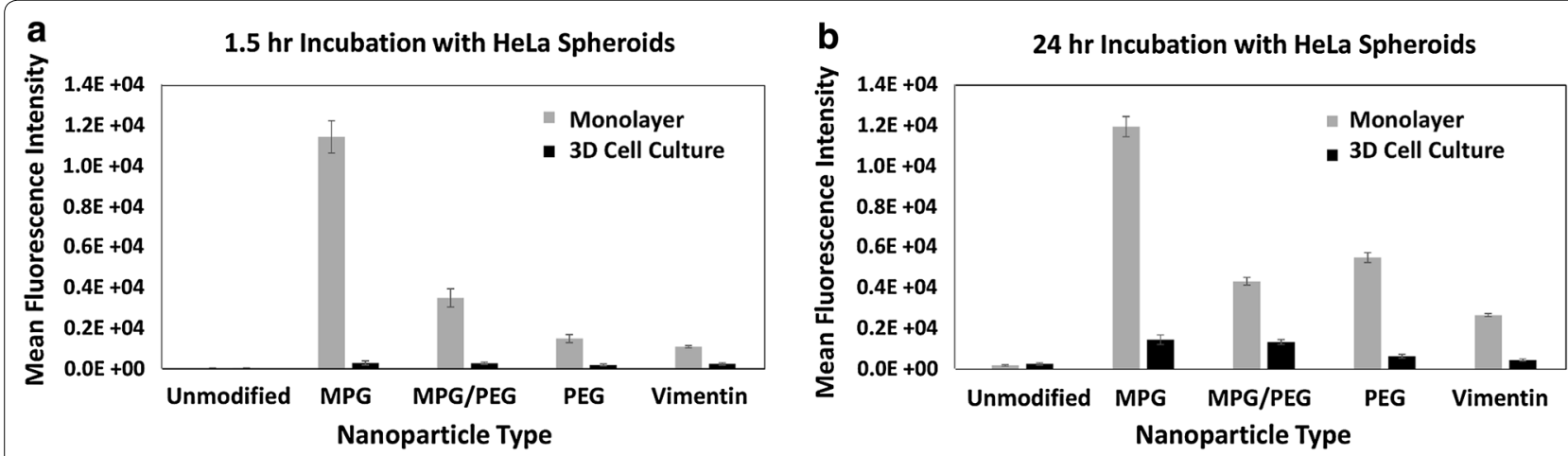

Fig. 6 Comparison of NP cellular internalization between HeLa monolayer and spheroid cell cultures. Results are shown at (a) 1.5 and (b) $24 \mathrm{~h}$ 


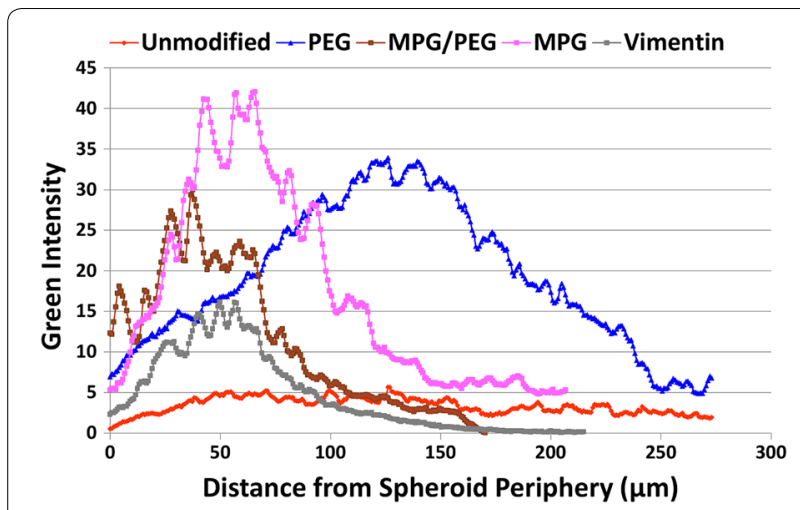

Fig. $7 \mathrm{NP}$ penetration into 3D cell culture (HeLa spheroids) after $1.5 \mathrm{~h}$ incubation

PEG, MPG/PEG, and VIM NPs, respectively, while PEG NPs penetrated $2 \times$ farther $(250 \mu \mathrm{m})$ into the spheroid than the MPG NPs. In comparison, unmodified NPs exhibited the lowest detection and penetration into the tumor spheroids. Representative images for each NP type are shown in Additional file 1: Figure S4.

\section{Discussion}

Building upon our previous work using avidin-palmitate PLGA NPs with modified surface chemistry [31], here we study the effects of contrasting and hybrid surface modifications on NP transport and cellular internalization in cervical tumor cells. Previously, avidin-palmitate NPs conjugated with the cell-penetrating peptide MPG were shown to have rapid internalization in monolayer cervical carcinoma (HeLa) cells, with a significant improvement over unmodified NPs. Here, we expand the suite of NP surface modifications to include PEG, VIM, and a hybrid modification of MPG and PEG. These formulations were evaluated for their internalization over short and extended time periods ( 1.5 and $24 \mathrm{~h}$, respectively) in monolayer cell culture, representing optimal transport conditions, and in tumor spheroid cultures, representing hypo-vascularized tumor lesions with diffusive transport limitations. Additionally, we extend our analysis beyond cancerous cells to include normal vaginal cells (VK2) to assess potential differences in the efficacy of different surface modifications when targeting tumor versus nontumor cells present in the female reproductive tract.

The NP formulations were characterized using standard methods to evaluate size, hydrodynamic size, and zeta potential (Additional file 1: Figure S1; Table S1). Unmodified NPs exhibited the most negative surface charge, while surface modification increased the surface potential to more positive values. MPG NPs exhibited the greatest increase in surface potential, as MPG is an amphipathic peptide that contains a cationic sequence known to enhance cell surface interaction. All NP surface modifications yielded an improvement in cellular association and internalization over unmodified NPs under optimal transport conditions in HeLa monolayer cell culture (Fig. 2a, b). MPG-modified NPs had the highest cellular association and internalization at both 1.5 and $24 \mathrm{~h}$, indicating their potential to efficaciously promote NP uptake. The hybrid MPG/PEG NPs showed significantly enhanced uptake at $1.5 \mathrm{~h}$, greater than both PEG and VIM-modified NPs. However, PEG significantly increased cellular internalization after a longer exposure time and surpassed the hybrid MPG/PEG NPs. The MPG, VIM, and hybrid NPs showed modest improvement in internalization after longer treatment duration. These results are in agreement with our previous work that MPG-modified NPs have a rapid mechanism of uptake in HeLa cells [31] while PEG-modified NPs take longer to internalize. This is consistent with PEG functionality, which is designed to prolong half-life in vivo.

In contrast, in normal vaginal cells (VK2), PEG-modified NPs exhibited similar high uptake and internalization at 1.5 and $24 \mathrm{~h}$ (Fig. 2c, d), while MPG and MPG/ PEG NPs showed only slight internalization. In fact, the MPG-modified NPs demonstrated greater than threefold improvement in internalization by cancerous relative to normal cells. These results suggest that the association and uptake of these NPs in normal cells may be mediated by different molecular mechanisms or cell surface moieties than in cancerous cells, which may have significant implications in more specific targeting of cancer lesions. The results further suggest that while VIM NPs are more efficacious than unmodified NPs, VIM most likely does not serve a specific targeting purpose for these cells. Enhancement is most likely due to the slightly more positive surface potential compared to that of the unmodified NPs. The high internalization of PEG-coated NPs in the VK2 cells, and also after $24 \mathrm{~h}$ in HeLa cells, indicates that these NPs might provide a means to enhance transport and concentration of therapeutics into normal vaginal tissue, while also offering the potential for enhanced uptake in cancerous cells.

Following the assessment of cellular internalization in optimally diffusive (monolayer) conditions, a similar evaluation was performed in 3D cell culture of HeLa cells to assess the transport and uptake of these NPs in hypovascularized tumor tissue. After the short incubation of $1.5 \mathrm{~h}$, all surface-modified NPs performed similarly (Fig. 5), demonstrating slightly increased internalization relative to the unmodified NPs. After $24 \mathrm{~h}$, the MPG and MPG/PEG NPs exhibited the most internalization relative to the other NP groups. Unlike the monolayer cultures, none of the groups saturated the spheroids at 
$1.5 \mathrm{~h}$, and instead elicited a more gradual, yet significant increase in internalization with longer exposure time. These results highlight the diffusive transport limitation through the 3D tissue culture as a time-dependent process, and enable a more detailed assessment of NP performance. The transport limitation in $3 \mathrm{D}$ tissue is well known (e.g., [52-56]), and derives from the extracellular matrix as well as cell-matrix and cell-cell interactions (such as E-cadherin) impeding diffusive flow [57, 58]. Whereas in monolayer HeLa and VK2 cell cultures the MPG and PEG NPs were internalized the most compared to the other groups, in the 3D cell culture the MPG and MPG/PEG NPs showed highest internalization. The diffusive transport limitation is further emphasized in Fig. 6, which compares the 2D and 3D internalization of the NPs in monolayer versus spheroid cultures.

As shown in Fig. 7, PEG NPs have the greatest depth of penetration into the spheroid tissue, with maximal concentration reached at $125 \mu \mathrm{m}$. In contrast, the MPG NPs achieved the highest concentration along the tumor periphery (maximally at $65 \mu \mathrm{m}$ ), but were unable to fully penetrate it. As shown in the monolayer, MPG NPs have the highest rate of cellular internalization, which is critical to the success of some biological therapeutics that cannot transverse cellular membranes alone, such as oligonucleotides, but this increased internalization may limit the depth of penetration. In contrast, the PEG modification, being very hydrophilic and less positively charged, enables NPs to more easily navigate the extracellular space and penetrate deeper into the spheroid tissue at the expense of internalization.

In living subjects, circulating NPs can preferentially lodge in tumor tissue by extravasating from fenestrated capillaries [59] as a result of the enhanced permeability and retention effect (EPR). Nevertheless, it has been shown that NP diffusive transport only extends about $30-50 \mu \mathrm{m}$ away from the vessels $[60,61]$. Consequently, therapeutics released from these NPs may not attain cytotoxic concentrations and also fail to affect quiescent (non-cycling) cells in regions distal to the vasculature [62]. Previously, we evaluated drug and NP transport in 2D and 3D cell culture, showing that nanoparticles layered with combinations of hexadecanethiol, phosphatidylcholine and high-density lipoprotein diffused deeper than PEGylated NPs into 3D cell culture representing hypo-vascularized tissue [63]. The NPs were uptaken in solid tumor tissue in vivo [64], and elicited distinctive drug release kinetics when loaded with paclitaxel or cisplatin [65]. Cytotoxicity experiments with free drug showed a substantial differential between 2D and 3D cell cultures, highlighting the increased resistance due to diffusive transport [34], although the drug-loaded layered NPs were substantially more efficacious in 3D cell culture than free-drug. While these findings are consistent with previous work showing decreased efficacy in 3D cell culture compared to monolayer $[32,60]$, they offer hope that modification of NPs to enhance their uptake and transport in cancerous tissue can help overcome the limitations of diffusive transport.

\section{Conclusions}

The results of this study suggest the potential usefulness of combination treatment employing a blend of different NP formulations to achieve internalization of therapeutics in cancerous tissue, e.g., in which PEG NPs are used for deep penetration into tissue distal from vasculature while MPG particles target proliferative regions close to blood vessels. Pathological examination of biopsy tissue could help to inform this approach. Interestingly, while the hybrid MPG/PEG NPs had similarly high uptake as MPG after $24 \mathrm{~h}$ in $3 \mathrm{D}$ cell culture, this group did not benefit from the combination of the cell penetrating and stealth attributes, and actually had a lower uptake and penetration depth than either MPG or PEG alone. In this case, internalization and diffusion may be competitive and thus inhibit overall NP uptake and transport. These results highlight the well-known need for a proper balance of NP properties to achieve both adequate internalization and diffusion, and emphasize that NP delivery assessment would benefit not only from consideration of different time points but also from evaluation of diffusive transport.

Future studies could potentially assess in further detail the time-dependent uptake of each NP group in monolayer as well as in 3D cell culture. The delivery of therapeutic agents (drug, siRNA, proteins) could be studied to assess efficacy. Evaluation in vivo would help establish NP distribution and clearance, as well as systemic toxicity. Additionally these studies would benefit from experimental as well as computational analysis [34, 42, 44, 66-69] to elucidate the complex interactions between NP parameters (surface modifications and transport), cell types (tumorigenic vs. normal), and the tumor microenvironment (cellular proliferation and death as a function of heterogeneous oxygen, nutrients, NP, and drug concentrations).

\section{Methods}

\section{Synthesis of avidin-palmitate conjugates}

Avidin-palmitate was conjugated to NP surfaces as previously described $[31,70,71]$ for subsequent reaction with biotinylated ligands: MPG, PEG, VIM, and an equimolar combination of MPG/PEG. Briefly, $40 \mathrm{mg}$ of avidin was dissolved in $4.8 \mathrm{~mL}$ of $2 \%$ sodium deoxycholate $(\mathrm{NaDC})$ in phosphate buffered saline (PBS) warmed to $37{ }^{\circ} \mathrm{C}$. Palmitic acid-N- hydroxysuccinimide ester (PA-NHS, 
Sigma) was dissolved in $2 \% \mathrm{NaDC}$ at $1 \mathrm{mg} / \mathrm{mL}$ and sonicated until well-mixed. $3.2 \mathrm{~mL}$ of the $1 \mathrm{mg} / \mathrm{mL}$ PA-NHS solution was added dropwise to the reaction vial, and reacted overnight at $37^{\circ} \mathrm{C}$. The following day, the reaction was dialyzed overnight in $1200 \mathrm{~mL}$ of $0.15 \% \mathrm{NaDC}$ in PBS heated to $37^{\circ} \mathrm{C}$. Free PA-NHS was removed using 3500 molecular weight cut off (MWCO) dialysis tubing, and the dialysis cassette contents were subsequently transferred to a vial and stored at $4{ }^{\circ} \mathrm{C}$.

\section{Nanoparticle synthesis}

We synthesized and characterized PLGA NPs encapsulating a fluorescent dye, Coumarin 6 (C6), to evaluate tumor penetration and distribution via fluorescence microscopy. From earlier studies [72-75] as well as our previous experiments, we have observed that negligible quantities $(\sim 1 \%)$ of $\mathrm{C} 6$ are released from NPs. This is attributed to the hydrophobic nature of $\mathrm{C} 6$ encapsulated within hydrophobic NPs. Therefore, C6 detected in cells reflects NP distribution in or on the cells, not C6 release and distribution. C6 NPs were synthesized as previously described using an oil-in-water $(\mathrm{o} / \mathrm{w})$ single emulsion technique [31, 71]. Briefly, C6 was encapsulated into 100-200 mg PLGA carboxyl-terminated polymer (0.55$0.75 \mathrm{dL} / \mathrm{g}$, LACTEL $\left.^{\circledR}\right)$. C6 was dissolved in methylene chloride (DCM) overnight at a concentration of $15 \mu \mathrm{g}$ C6 per mg of PLGA. The following day, the solution was added dropwise to a $5 \%$ polyvinyl alcohol (PVA) solution of equal volume, vortexed and sonicated. The resulting NPs were hardened during solvent evaporation for $3 \mathrm{~h}$. For unmodified NPs, the NPs were washed after hardening, and centrifuged 3 times at $4{ }^{\circ} \mathrm{C}$ in deionized water $\left(\mathrm{diH}_{2} \mathrm{O}\right)$ to remove residual solvent. For avidin-palmitate surface-modified NPs, a similar protocol was followed $[31,71,73]$. NP formulations were synthesized by adding $(1 \mathrm{mg} / \mathrm{mL})$ avidin-palmitate to the $5 \%$ PVA solution. NPs were collected after the first wash and incubated for 30 min with biotinylated ligands at a molar ratio of 3:1 ligand:avidin in PBS. After conjugation, the NPs were washed two more times with $\operatorname{diH}_{2} \mathrm{O}$ by centrifugation and subsequent washing. All NPs were frozen, lyophilized, and stored at $-20^{\circ} \mathrm{C}$ until use.

\section{Cell culture}

VK2/E6E7 vaginal epithelial (VK2) and human cervical carcinoma (HeLa) immortalized cell lines were kindly provided by Dr. Kenneth Palmer's lab (University of Louisville). These cell lines were obtained and authenticated through ATCC. We selected the VK2/E6E7 cell line, since for intravaginal delivery these would be the first "normal" cells to encounter NP treatment. HeLa cells provide the ability to assess NP behavior against a cervical cancer cell line in vitro. VK2/E6E7 vaginal epithelial cells (VK2) were maintained in Defined Kerotinocyte-Serum Free medium (SFM) supplemented with Defined Keratinocyte-SFM Growth Supplement. HeLa cells were maintained in minimal essential media (MEM) supplemented with $10 \%$ fetal bovine serum and $1 \%$ penicillin-streptomycin in standard culture conditions. Cell media was checked and changed daily.

\section{Tumor spheroid formation}

HeLa cells were used for tumor spheroid formation. Cells were grown to $80 \%$ confluence before harvesting. Twenty-four well tissue culture plates (Corning) were coated with a $1 \%(\mathrm{w} / \mathrm{v})$ agarose gel $24 \mathrm{~h}$ before spheroid formation to prevent cell adherence. For formation, 100,000 cells were placed in each well and lightly shaken (100 rpm) for $15 \mathrm{~min}$ on a reciprocating shaker. After 7-14 days of incubation, spheroid formation occurred by self-aggregation to sizes ranging from 500 to $1000 \mu \mathrm{m}$.

\section{Monolayer (2D) cellular uptake and microscopy}

2D cellular imaging of 1.5 and $24 \mathrm{~h}$ NP uptake and penetration in HeLa and VK2 cells was performed via inverted epifluorescence microscopy [31]. VK2 and HeLa cells were seeded $24 \mathrm{~h}$ prior to NP administration in LabTek 8-well chamber slides at a density of 50,000 and 40,000 cells per well for 1.5 and $24 \mathrm{~h}$ uptake, respectively. For NP administration, NPs were massed out and dissolved to reach a final stock concentration of $0.6 \mathrm{mg} / \mathrm{mL}$ in PBS++ (containing $\mathrm{CaCl}_{2}$ and $\mathrm{MgCl}_{2}$ ) to aid cell adherence. One hundred microliters of fresh media were added to the cells, and NPs were then added to obtain a final NP concentration of $200 \mu \mathrm{g} / \mathrm{mL}$.

After either 1.5 or $24 \mathrm{~h}$ incubation, cells were washed five times in $0.5 \mathrm{~mL}$ of $1 \mathrm{X}$ PBS to remove any unbound or non-internalized NPs. Cells were then fixed with $0.3 \mathrm{~mL}$ of $4 \%$ paraformaldehyde and incubated for $10 \mathrm{~min}$ at room temperature (RT). Cells were subsequently washed twice with $0.5 \mathrm{~mL}$ of PBS and permeabilized with $0.3 \mathrm{~mL}$ of $0.1 \%$ Triton $\mathrm{X}-100$ in $1 \%$ bovine serum albumin (BSA) $\mathrm{PBS}++$ for $10 \mathrm{~min}$ at RT. After permeabilization, cells were incubated with $0.3 \mathrm{~mL}$ of 1:40 Texas Red Phalloidin in $1 \%$ BSA PBS++ for $20 \mathrm{~min}$ at RT for cytoskeleton staining and were subsequently washed twice with $0.5 \mathrm{~mL}$ PBS. Cells were then incubated with $0.3 \mathrm{~mL}$ of $4 \mu \mathrm{g} / \mathrm{mL}$ Hoechst in $1 \%$ BSA PBS ++ for $10 \mathrm{~min}$ at $37^{\circ} \mathrm{C}$ for nuclear staining. Finally, cells were washed twice in PBS and once in $\operatorname{diH}_{2} \mathrm{O}$, then mounted in Vectashield non-hardening mounting medium (Vector Laboratories, VWR) and kept at $4{ }^{\circ} \mathrm{C}$ until imaged [31].

Inverted epifluorescence microscopy was utilized to assess cellular uptake of NPs in 2D monolayers. Briefly, cells were prepared as described above and imaged in 8-well LabTek chamber slides using the following filter 
settings: 4',6-diamidino-2-phenylindole (DAPI) to visualize Hoechst, green fluorescent protein (GFP) for C6, and the Texas Red channel to evaluate NP uptake in 2D. Exposure times for DAPI, GFP, and Texas Red were kept consistent throughout experiments and were as follows: DAPI at $45 \mathrm{~ms}$ (excitation/emissions: $358 / 461 \mathrm{~nm}$ ); GFP at $60 \mathrm{~ms}(593 / 615 \mathrm{~nm})$; and Texas Red at $180 \mathrm{~ms}$ $(488 / 515 \mathrm{~nm})$.

\section{Spheroid (3D) cellular uptake and microscopy}

To assess the differences in NP uptake and distribution through hypo-vascularized tumor tissue, HeLa spheroids were incubated with $0.01 \mathrm{mg} / \mathrm{mL}$ of NPs and visualized using confocal microscopy. Diffusion profiles through the spheroids for each NP formulation were evaluated by quantifying the fluorescence intensity in z-stack images as a function of distance from the spheroid periphery. After NP administration, tumor spheroids were transferred to LabTek 8-well chamber slides for fluorescent staining and were washed five times with $0.2 \mathrm{~mL} 1 \mathrm{X}$ PBS. Tumor spheroids were subsequently incubated for $10 \mathrm{~min}$ at RT with $0.2 \mathrm{~mL} 4 \%$ paraformaldehyde for spheroid fixation. Following fixation, spheroids were washed twice with $0.2 \mathrm{~mL}$ of PBS, followed by incubation with $0.2 \mathrm{~mL}$ of 1:40 Texas Red Phalloidin in 1\% BSA PBS++ for $20 \mathrm{~min}$ at RT for cytoskeleton staining. Spheroids were then washed twice with $0.2 \mathrm{~mL}$ PBS and incubated with $0.2 \mathrm{~mL}$ of $4 \mu \mathrm{g} / \mathrm{mL}$ Hoechst in $1 \%$ BSA PBS++ for $10 \mathrm{~min}$ at $37{ }^{\circ} \mathrm{C}$ for nuclear staining. Finally, spheroids were washed twice in $0.2 \mathrm{~mL}$ PBS and once in $0.2 \mathrm{~mL}$ $\mathrm{diH}_{2} \mathrm{O}$. Spheroids were then mounted with Vectashield non-hardening mounting medium. 3D uptake and distribution of NPs through tumor spheroids was assessed using confocal microscopy. Images were processed using ImageJ by taking representative samples from tumor cross-sections ( $\geq 90$ averaged profiles for each sample).

\section{Flow cytometry analysis}

Cells were plated in 6-well plates (Corning) at a density of 200,000 cells per well. Both VK2 and HeLa cell lines were incubated with NP suspensions at $0.05 \mathrm{mg} / \mathrm{mL}$ for either 1.5 or $24 \mathrm{~h}$ in a $37{ }^{\circ} \mathrm{C}$ humidified chamber in the appropriate medium [31]. An unmodified NP control group was used to compare uptake relative to surfacemodified NPs, as our previous work demonstrated minimal difference between unmodified and avidin-modified NP association and uptake [31]. After incubation, the cells were washed five times with $\mathrm{PBS}++$. Next, the cells were dissociated with enzyme-free cell dissociation buffer (ThermoFisher). The dissociated cells were moved to FACS tubes, centrifuged, and resuspended in a FACS buffer solution containing $1 \%$ BSA and $0.1 \%$ sodium azide. From each sample, half the cells were moved to separate FACS tubes and kept on ice until analyzed (total associated samples). The remaining cells were exposed to $0.4 \%$ trypan blue for 5 min to quench extracellular fluorescence, washed twice in FACS buffer, and kept on ice until analyzed (internalized samples) [31]. All FACS tubes from both groups were analyzed using a BD LSRFortessa Flow Cytometer (BD Biosciences). Data were analyzed using FlowJo software (FlowJo Enterprise), and a minimum of 10,000 cells were analyzed per sample. For flow cytometry analysis of tumor spheroids, a similar protocol was followed using spheroids after 7 days of growth. Instead of enzyme-free cell dissociation buffer, $0.25 \%$ Trypsin-EDTA was used to fully disaggregate the spheroids before centrifugation and resuspension in FACS buffer solution.

\section{Statistical analysis}

Experiments were conducted each with a minimum sample size of $\mathrm{n}=3$. Data were analyzed by applying Tukey's test with significance $\mathrm{p}<0.05$. Unless otherwise noted, all figure error bars represent the standard deviation of the measurements. To enable clear interpretation, statistically similar results are shown linked with an overbar in the figures of the "Results" section.

\section{Additional file}

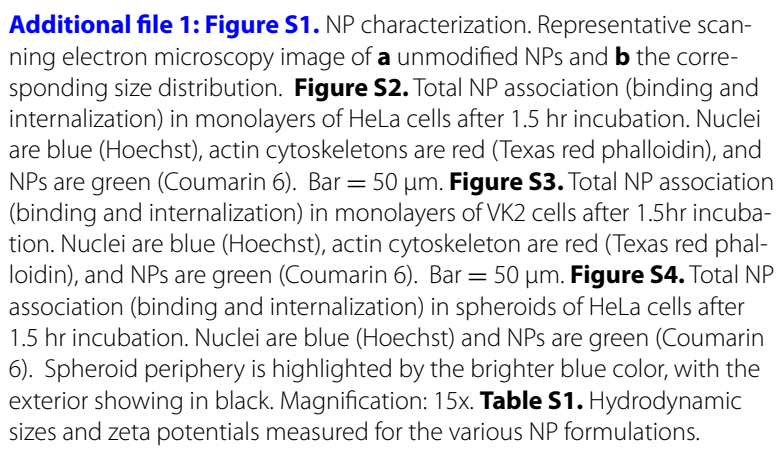

Additional file 1: Figure S1. NP characterization. Representative scanning electron microscopy image of $\mathbf{a}$ unmodified NPs and $\mathbf{b}$ the corresponding size distribution. Figure S2. Total NP association (binding and internalization) in monolayers of HeLa cells after 1.5 hr incubation. Nuclei are blue (Hoechst), actin cytoskeletons are red (Texas red phalloidin), and NPs are green (Coumarin 6). Bar $=50 \mu \mathrm{m}$. Figure S3. Total NP association (binding and internalization) in monolayers of VK2 cells after $1.5 \mathrm{hr}$ incubation. Nuclei are blue (Hoechst), actin cytoskeleton are red (Texas red phalloidin), and NPs are green (Coumarin 6). Bar $=50 \mu \mathrm{m}$. Figure S4. Total NP association (binding and internalization) in spheroids of HeLa cells after $1.5 \mathrm{hr}$ incubation. Nuclei are blue (Hoechst) and NPs are green (Coumarin 6). Spheroid periphery is highlighted by the brighter blue color, with the exterior showing in black. Magnification: 15x. Table S1. Hydrodynamic sizes and zeta potentials measured for the various NP formulations.

\section{Abbreviations}

2D: two-dimensional; 3D: three-dimensional; BSA: bovine serum albumin; C6: Coumarin 6; DAPI: 4',6-diamidino-2-phenylindole; $\mathrm{diH}_{2} \mathrm{O}$ : deionized water; FACS: Fluorescence Activated Cell Sorting; GFP: green fluorescent protein; MWCO: molecular weight cut off; NaDC: sodium deoxycholate; NP: nanoparticle; PA-NHS: palmitic acid-N-hydroxysuccinimide ester; PBS: phosphate buffered saline; PEG: polyethylene glycol; PLGA: poly(lactic-co-glycolic) acid; PVA: $5 \%$ polyvinyl alcohol; RT: room temperature; SEM: scanning electron microscopy; VIM: Vimentin tubulin binding site peptide.

\section{Authors' contributions}

LBS performed the experiments and drafted the manuscript. LTC helped to perform the experiments and draft the manuscript, and performed the statistical analysis. HBF conceived of the study, participated in its design and coordination, and drafted the manuscript. JSR conceived of the study, participated in its design and coordination, and drafted the manuscript. All authors read and approved the final manuscript. 


\begin{abstract}
Author details
${ }^{1}$ Department of Bioengineering, University of Louisville, 505 S. Hancock, CTRB 623, Louisville, KY 40208, USA. ${ }^{2}$ James Graham Brown Cancer Center, University of Louisville, Louisville, KY, USA. ${ }^{3}$ Department of Pharmacology and Toxicology, University of Louisville, Louisville, KY, USA. ${ }^{4}$ Department of Microbiology and Immunology, University of Louisville, Louisville, KY, USA. ${ }^{5}$ Center for Predictive Medicine, University of Louisville, Louisville, KY, USA.
\end{abstract}

\section{Acknowledgements}

HBF acknowledges partial support from NIH/NCI U54CA143907.

\section{Competing interests}

The authors declare that they have no competing interests.

Received: 28 January 2016 Accepted: 12 April 2016

Published online: 22 April 2016

\section{References}

1. ACS. Learn About Cancer-Cervical Cancer-Detailed Guide-What are the key statistics about cervical cancer? American Cancer Society. 2016. http://www.cancer.org/cancer/cervicalcancer/detailedguide/ cervical-cancer-key-statistics.

2. Siegel R, Ward E, Brawley O, Jemal A. Cancer statistics, 2011: the impact of eliminating socioeconomic and racial disparities on premature cancer deaths. CA Cancer J Clin. 2011;61(4):212-36. doi:10.3322/caac.20121.

3. CDC. Gynecologic cancers. Center for Disease Control (CDC). 2015. http:// www.cdc.gov/cancer/cervical/.

4. NCI. NCl: HPV and cancer. National Cancer Institute. 2015. http://www.cancer. gov/about-cancer/causes-prevention/risk/infectious-agents/hpv-fact-sheet.

5. Schiller JT, Castellsague X, Garland SM. A review of clinical trials of Human papillomavirus prophylactic vaccines. Vaccine. 2012;30:F123-38. doi:10.1016/j.vaccine.2012.04.108.

6. Hildesheim A, Herrero R, Wacholder S, Rodriguez AC, Solomon D, Bratti MC, et al. Effect of human papillomavirus 16/18 L1 viruslike particle vaccine among young women with preexisting infection - a randomized trial. JAMA. 2007;298(7):743-53. doi:10.1001/jama.298.7.743.

7. Chen JZ, Gu WY, Yang L, Chen C, Shao RF, Xu KW, et al. Nanotechnology in the management of cervical cancer. Rev Med Virol. 2015;25:72-83. doi:10.1002/rmv.1825.

8. Jensen PT, Groenvold M, Klee MC, Thranov I, Petersen MA, Machin D. Early-stage cervical carcinoma, radical hysterectomy, and sexual function. A longitudinal study. Cancer. 2004;100(1):97-106. doi:10.1002/ cncr.11877.

9. Kyrgiou M, Koliopoulos G, Martin-Hirsch P, Arbyn M, Prendiville W, Paraskevaidis E. Obstetric outcomes after conservative treatment for intraepithelial or early invasive cervical lesions: systematic review and meta-analysis. Lancet. 2006;367(9509):489-98. doi:10.1016/S0140-6736(06)68181-6.

10. Yang M, Yu T, Wang YY, Lai SK, Zeng Q, Miao BL, et al. Vaginal delivery of paclitaxel via nanoparticles with non-mucoadhesive surfaces suppresses cervical tumor growth. Adv Healthc Mat. 2014;3(7):1044-52. doi:10.1002/ adhm.201300519.

11. Bertrand N, Wu J, Xu X, Kamaly N, Farokhzad OC. Cancer nanotechnology: the impact of passive and active targeting in the era of modern cancer biology. Adv Drug Deliv Rev. 2014;66:2-25. doi:10.1016/j. addr.2013.11.009.

12. Danhier F, Ansorena E, Silva JM, Coco R, Le Breton A, Preat V. PLGA-based nanoparticles: an overview of biomedical applications. J Control Release. 2012;161(2):505-22. doi:10.1016/j.jconrel.2012.01.043.

13. Soppimath KS, Aminabhavi TM, Kulkarni AR, Rudzinski WE. Biodegradable polymeric nanoparticles as drug delivery devices. J Control Release. 2001;70(1-2):1-20.

14. Dawidczyk CM, Kim C, Park JH, Russell LM, Lee KH, Pomper MG, et al. State-of-the-art in design rules for drug delivery platforms: lessons learned from FDA-approved nanomedicines. J Control Release. 2014;187:133-44. doi:10.1016/j.jconrel.2014.05.036.

15. Salmaso S, Caliceti P. Stealth properties to improve therapeutic efficacy of drug nanocarriers. J Drug Deliv. 2013;2013:374252. doi:10.1155/2013/374252
16. Amoozgar Z, Yeo Y. Recent advances in stealth coating of nanoparticle drug delivery systems. Wiley Interdiscip Rev Nanomed Nanobiotechnol. 2012:4(2):219-33. doi:10.1002/wnan.1157.

17. Alexander-Bryant AA, Vanden Berg-Foels WS, Wen X. Bioengineering strategies for designing targeted cancer therapies. Adv Cancer Res. 2013;118:1-59. doi:10.1016/B978-0-12-407173-5.00002-9.

18. Erazo-Oliveras A, Muthukrishnan N, Baker R, Wang TY, Pellois JP. Improving the endosomal escape of cell-penetrating peptides and their cargos: strategies and challenges. Pharmaceuticals (Basel). 2012;5(11):1177-209. doi:10.3390/ph5111177

19. Koren E, Torchilin VP. Cell-penetrating peptides: breaking through to the other side. Trends Mol Med. 2012;18(7):385-93. doi:10.1016/j. molmed.2012.04.012

20. Lehto T, Kurrikoff K, Langel U. Cell-penetrating peptides for the delivery of nucleic acids. Expert Opin Drug Deliv. 2012;9(7):823-36. doi:10.1517/1742 5247.2012 .689285$.

21. Margus H, Padari K, Pooga M. Cell-penetrating peptides as versatile vehicles for oligonucleotide delivery. Mol Ther. 2012;20(3):525-33. doi:10.1038/mt.2011.284.

22. Meade BR, Dowdy SF. Exogenous siRNA delivery using peptide transduction domains/cell penetrating peptides. Adv Drug Deliv Rev. 2007;59(23):134-40. doi:10.1016/j.addr.2007.03.004.

23. Meade BR, Dowdy SF. Enhancing the cellular uptake of siRNA duplexes following noncovalent packaging with protein transduction domain peptides. Adv Drug Deliv Rev. 2008;60(4-5):530-6.

24. Morris MC, Deshayes S, Heitz F, Divita G. Cell-penetrating peptides: from molecular mechanisms to therapeutics. Biol Cell. 2008;100(4):201-17. doi:10.1042/BC20070116.

25. Mussbach F, Franke M, Zoch A, Schaefer B, Reissmann S. Transduction of peptides and proteins into live cells by cell penetrating peptides. J Cell Biochem. 2011:112(12):3824-33. doi:10.1002/jcb.23313.

26. Henriques ST, Melo MN, Castanho MA. Cell-penetrating peptides and antimicrobial peptides: how different are they? Biochem J. 2006;399(1):17. doi:10.1042/BJ20061100.

27. Raucher D, Ryu JS. Cell-penetrating peptides: strategies for anticancer treatment. Trends Mol Med. 2015;21(9):560-70. doi:10.1016/j. molmed.2015.06.005

28. Gupta B, Levchenko TS, Torchilin VP. Intracellular delivery of large molecules and small particles by cell-penetrating proteins and peptides. Adv Drug Deliv Rev. 2005;57(4):637-51. doi:10.1016/j.addr.2004.10.007.

29. Mokhtarieh AA, Kim S, Lee Y, Chung BH, Lee MK. Novel cell penetrating peptides with multiple motifs composed of RGD and its analogs. Biochem Biophys Res Commun. 2013;432(2):359-64. doi:10.1016/j. bbrc.2013.01.096

30. Simeoni F, Morris MC, Heitz F, Divita G. Insight into the mechanism of the peptide-based gene delivery system MPG: implications for delivery of siRNA into mammalian cells. Nucleic Acids Res. 2003;31(11):2717-24.

31. Steinbach JM, Seo YE, Saltzman WM. Cell penetrating peptide-modified PLGA nanoparticles with enhanced cell internalization. Acta Biomater. 2016;15(30):49-61.

32. Minchinton Al, Tannock IF. Drug penetration in solid tumours. Nat Rev Cancer. 2006;6(8):583-92. doi:10.1038/nrc1893.

33. Sutherland R, McCredie J, Inch W. Growth of multicell spheroids in tissue culture as a model of nodular carcinomas. J Natl Cancer Inst. 1971;46:113-20.

34. Curtis LT, England CG, Wu M, Lowengrub J, Frieboes HB. An interdisciplinary computational/experimental approach to evaluate drugloaded gold nanoparticle tumor cytotoxicity. Nanomedicine (Lond). 2016;11(3):197-216. doi:10.2217/nnm.15.195.

35. Curtis LT, Wu M, Lowengrub J, Decuzzi P, Frieboes HB. Computational modeling of tumor response to drug release from vasculature-bound nanoparticles. PLoS ONE. 2015;10(12):e0144888. doi:10.1371/journal. pone.0144888.

36. Goodman TT, Olive PL, Pun SH. Increased nanoparticle penetration in collagenase-treated multicellular spheroids. Int J Nanomed. 2007;2(2):265-74

37. Huang K, Ma H, Liu J, Huo S, Kumar A, Wei T, et al. Size-dependent localization and penetration of ultrasmall gold nanoparticles in cancer cells, multicellular spheroids, and tumors in vivo. ACS Nano. 2012;6(5):4483-93. doi:10.1021/nn301282m 
38. Neshatian M, Chung S, Yohan D, Yang C, Chithrani DB. Uptake of gold nanoparticles in breathless (hypoxic) cancer cells. J Biomed Nanotechnol. 2015;11(7):1162-72.

39. Frieboes HB, Wu M, Lowengrub J, Decuzzi P, Cristini V. A computational model for predicting nanoparticle accumulation in tumor vasculature. PLOS ONE. 2013;8(2):e56876. doi:10.1371/journal.pone.0056876.

40. Wu M, Frieboes HB, Chaplain MA, McDougall SR, Cristini V, Lowengrub JS. The effect of interstitial pressure on therapeutic agent transport: coupling with the tumor blood and lymphatic vascular systems. J Theor Biol. 2014;355:194-207. doi:10.1016/j.jtbi.2014.04.012.

41. Li M, Panagi Z, Avgoustakis K, Reineke J. Physiologically based pharmacokinetic modeling of PLGA nanoparticles with varied mPEG content. Int J Nanomed. 2012;7:1345-56. doi:10.2147/IJN.S23758.

42. Gao Y, Li M, Chen B, Shen Z, Guo P, Wientjes MG, et al. Predictive models of diffusive nanoparticle transport in 3-dimensional tumor cell spheroids. The AAPS journal. 2013;15(3):816-31. doi:10.1208/s12248-013-9478-2.

43. Decuzzi P, Godin B, Tanaka T, Lee SY, Chiappini C, Liu X, et al. Size and shape effects in the biodistribution of intravascularly injected particles. J Control Release. 2010;141(3):320-7. doi:10.1016/j.jconrel.2009.10.014.

44. Godin B, Driessen WH, Proneth B, Lee SY, Srinivasan S, Rumbaut R, et al. An integrated approach for the rational design of nanovectors for biomedical imaging and therapy. Adv Genet. 2010;69:31-64. doi:10.1016/ S0065-2660(10)69009-8.

45. Boso DP, Lee SY, Ferrari M, Schrefler BA, Decuzzi P. Optimizing particle size for targeting diseased microvasculature: from experiments to artificial neural networks. Int J Nanomed. 2011;6:1517-26. doi:10.2147/IJN.S20283.

46. Decuzzi P, Ferrari $M$. The adhesive strength of non-spherical particles mediated by specific interactions. Biomaterials. 2006;27(30):5307-14. doi:10.1016/j.biomaterials.2006.05.024.

47. Decuzzi P, Pasqualini R, Arap W, Ferrari M. Intravascular delivery of particulate systems: does geometry really matter? Pharm Res. 2009;26(1):235-43. doi:10.1007/s11095-008-9697-x.

48. Lee SY, Ferrari M, Decuzzi P. Shaping nano-/micro-particles for enhanced vascular interaction in laminar flows. Nanotechnology. 2009;20(49):495101. doi:10.1088/0957-4484/20/49/495101.

49. Curtis LT, Wu M, Lowengrub J, Decuzzi P, Frieboes HB. Computational modeling of tumor response to drug release from vasculature-bound nanoparticles. PLoS ONE. 2015;10(12):e0144888.

50. Balzeau J, Peterson A, Eyer J. The vimentin-tubulin binding site peptide (Vim-TBS.58-81) crosses the plasma membrane and enters the nuclei of human glioma cells. Int J Pharm. 2012;423(1):77-83. doi:10.1016/j. ijpharm.2011.04.067.

51. Saini A, Jaswal RR, Negi R, Nandel FS. Insights on the structural characteristics of Vim-TBS (58-81) peptide for future applications as a cell penetrating peptide. Biosci Trends. 2013;7(5):209-20. doi:10.5582/bst.2013. v7.5.209.

52. Polacheck WJ, Zervantonakis IK, Kamm RD. Tumor cell migration in complex microenvironments. Cell Mol Life Sci. 2013;70(8):1335-56. doi:10.1007/s00018-012-1115-1.

53. Tchafa AM, Shah AD, Wang S, Duong MT, Shieh AC. Three-dimensional cell culture model for measuring the effects of interstitial fluid flow on tumor cell invasion. J Vis Exp. 2012;. doi:10.3791/4159.

54. Yamada KM, Cukierman E. Modeling tissue morphogenesis and cancer in 3D. Cell. 2007;130(4):601-10. doi:10.1016/j.cell.2007.08.006.

55. Griffith LG, Swartz MA. Capturing complex 3D tissue physiology in vitro. Nat Rev Mol Cell Biol. 2006;7(3):211-24. doi:10.1038/nrm1858.

56. Lee J, Lilly GD, Doty RC, Podsiadlo P, Kotov NA. In vitro toxicity testing of nanoparticles in 3D cell culture. Small. 2009;5(10):1213-21. doi:10.1002/ smll.200801788.

57. Nyga A, Cheema U, Loizidou M. 3D tumour models: novel in vitro approaches to cancer studies. J Cell Commun Signal. 2011;5(3):239-48. doi:10.1007/s12079-011-0132-4.
58. Santini MT, Rainaldi G, Indovina PL. Apoptosis, cell adhesion and the extracellular matrix in the three-dimensional growth of multicellular tumor spheroids. Crit Rev Oncol Hematol. 2000;36(2-3):75-87.

59. Roberts WG, Palade GE. Increased microvascular permeability and endothelial fenestration induced by vascular endothelial growth factor. J Cell Sci. 1995;108(Pt 6):2369-79.

60. Primeau AJ, Rendon A, Hedley D, Lilge L, Tannock IF. The distribution of the anticancer drug Doxorubicin in relation to blood vessels in solid tumors. Clin Cancer Res. 2005;11(24 Pt 1):8782-8. doi:10.1158/1078-0432. CCR-05-1664.

61. Hait WN, Hambley TW. Targeted cancer therapeutics. Cancer Res. 2009;69(4):1263-7. doi:10.1158/0008-5472.CAN-08-3836 (discussion 7).

62. Warren KE. Novel therapeutic delivery approaches in development for pediatric gliomas. CNS Oncol. 2013;2(5):427-35. doi:10.2217/cns.13.37.

63. England CG, Priest T, Zhang G, Sun X, Patel DN, McNally LR, et al. Enhanced penetration into 3D cell culture using two and three layered gold nanoparticles. Int J Nanomed. 2013;8(1):3602-17.

64. England CG, Huang JS, James KT, Zhang G, Gobin AM, Frieboes HB. Detection of Phosphatidylcholine- Coated Gold Nanoparticles in Orthotopic Pancreatic Adenocarcinoma using Hyperspectral Imaging. PLoS ONE. 2015;10(6):e0129172. doi:10.1371/journal.pone.0129172.

65. England CG, Miller MC, Kuttan A, Trent JO, Frieboes HB. Release kinetics of paclitaxel and cisplatin from two and three layered gold nanoparticles. Eur J Pharm Biopharm. 2015;92:120-9. doi:10.1016/j.ejpb.2015.02.017.

66. Frieboes HB, Sinek JP, Nalcioglu O, Fruehauf JP, Cristini V. Nanotechnology in cancer drug therapy: a biocomputational approach. In: Ferrari M, Lee AP, Lee LJ, editors. BioMEMS and biomedical nanotechnology: volume I biological and biomedical nanotechnology. Boston: Springer Science + Business Media, LLC; 2006. p. 435-60.

67. Li M, Czyszczon EA, Reineke JJ. Delineating intracellular pharmacokinetics of paclitaxel delivered by PLGA nanoparticles. Drug Deliv Transl Res. 2013;3(6):551-61. doi:10.1007/s13346-013-0162-y.

68. van de Ven AL, Abdollahi B, Martinez CJ, Burey LA, Landis MD, Chang JC, et al. Modeling of nanotherapeutics delivery based on tumor perfusion. New J Phys. 2013;15:55004. doi:10.1088/1367-2630/15/5/055004.

69. van de Ven AL, Wu M, Lowengrub J, McDougall SR, Chaplain MA, Cristini $\checkmark$, et al. Integrated intravital microscopy and mathematical modeling to optimize nanotherapeutics delivery to tumors. AIP Adv. 2012;2(1):11208. doi:10.1063/1.3699060.

70. Fahmy TM, Samstein RM, Harness CC, Mark Saltzman W. Surface modification of biodegradable polyesters with fatty acid conjugates for improved drug targeting. Biomaterials. 2005;26(28):5727-36. doi:10.1016/j. biomaterials.2005.02.025.

71. Martin DT, Steinbach JM, Liu J, Shimizu S, Kaimakliotis HZ, Wheeler MA, et al. Surface-modified nanoparticles enhance transurothelial penetration and delivery of survivin siRNA in treating bladder cancer. Mol Cancer Ther. 2014;13(1):71-81. doi:10.1158/1535-7163.MCT-13-0502.

72. Woodrow KA, Cu Y, Booth CJ, Saucier-Sawyer JK, Wood MJ, Saltzman WM. Intravaginal gene silencing using biodegradable polymer nanoparticles densely loaded with small-interfering RNA. Nat Mater. 2009;8(6):526-33. doi:10.1038/nmat2444.

73. Cu Y, Booth CJ, Saltzman WM. In vivo distribution of surface-modified PLGA nanoparticles following intravaginal delivery. J Control Release. 2011;156(2):258-64. doi:10.1016/j.jconrel.2011.06.036.

74. Li J, Feng L, Fan L, Zha Y, Guo L, Zhang Q, et al. Targeting the brain with PEG-PLGA nanoparticles modified with phage-displayed peptides. Biomaterials. 2011;32(21):4943-50. doi:10.1016/j.biomaterials.2011.03.031.

75. Desai MP, Labhasetwar V, Walter E, Levy RJ, Amidon GL. The mechanism of uptake of biodegradable microparticles in Caco-2 cells is size dependent. Pharm Res. 1997;14(11):1568-73. 